\title{
Idiopathic Lumbosacral Spinal Chronic Subdural Hematoma
}

\author{
HyunJin Ma, Insoo Kim \\ Department of Neurosurgery, Dongsan Medical Center, Keimyung University College of Medicine, Daegu, Korea
}

Reports of spontaneous spinal chronic subdural hematoma (SDH) are extremely rare. In the limited cases reported, the origin, symptoms and treatments are diversely revealed. Therefore accurate diagnoses and proper treatments are difficult to determine. We report a 74-year-old male presented with severe low back pain and both buttocks pain. Magnetic resonance imaging showed a diffuse subdural infiltrating mass compress nerve roots at the level of L2-S2. Emergency operation for decompression and excision of mass revealed spinal chronic SDH. His pain was subsided after the operation. We report a rare case of idiopathic lumbosacral spinal chronic SDH and discuss various causes and treatments.

Key Words: Spinal subdural hematoma - Spinal cord compression

\section{INTRODUCTION}

Spinal cord hematomas are uncommon. Among spinal cord hematomas, subdural hematoma $(\mathrm{SDH})$ is an even more rare entity. Based on causes, spinal SDH is classified as idiopathic or secondary ${ }^{8,9)}$. Spinal SDH can be developed as a result of anticoagulation therapy, blood dyscrasia, spinal puncture, trauma, spinal anesthesia or vascular malformation ${ }^{1,7}$. Spinal $\mathrm{SDH}$ is classified as acute, subacute, chronic according to the course of clinical symptoms ${ }^{8)}$.

The symptoms of acute spinal SDH begin with severe and sudden onset back pain accompanied by severe radicular pain, and rapid progression to paraplegia within several days. The symptoms of subacute SDH begin with less well localized back, hip and leg pain, gradually progress to paraplegia over a period of 1 week. Reports of spontaneous spinal chronic SDH are extremely rare with only 23 cases $^{5}$. Spinal chronic SDH begins with the signs and symptoms of progressive spinal cord compression occurring over a period of several months to several years, with mild or absent pain. Spontaneous spinal SDH occurs most commonly in the lower thoracic or thoracolumbar lesions and develops during the sixth decade of life ${ }^{5,12)}$. For the best outcomes, early surgical decompression is commonly needed.

- Received: January 8, 2012 • Revised: March 24, 2012

- Accepted: March 26, 2012

Corresponding Author: Insoo Kim, MD, PhD

Department of Neurosurgery, Dongsan Medical Center, 56 Dalseong-ro, Jung-gu, Daegu 700-712, Korea

Tel: +82-53-250-7730, Fax: +82-53-250-7356

E-mail:neurokim@dsmc.or.kr
We report a case of spontaneous spinal chronic SDH located at lumbosacral region without underlying pathology.

\section{CASE REPORT}

A 74-year-old male was presented with low back pain and both buttocks pain for 16 days. The pain in both buttocks had gradually progressed to both legs in the L 5 dermatome and the intensity of pain was aggravated during the last 3 days. There were no history of trauma, infection or anticoagulant therapy. On admission, his vital signs were stable, and neurological examination revealed normal tone in upper limb and lower limb. Power in extremities were grade $5 / 5$ at all joints in Medical Research Council Muscle Strength Grading Scale ${ }^{2)}$.

The Plane X-ray films of lumbar spine showed multiple spur formation and intervertebral space narrowing at L5-S1 and some degenerative change. Lumbar spine magnetic resonance image revealed a diffuse subdural infiltrating mass compress nerve roots at the level of L2-S2. The mass presented an isoto-hyperintense signal on T1-weighted magnetic resonance imaging (MRI) to the spinal cord, and an iso-to-hypointense signal on T2-weighted MRI (Fig. 1).

As the patient complained of unbearable pain, we underwent an emergency surgical decompression with L4 and L5 laminotomy. There was no obvious extradural hematoma and abnormality but the dura mater was bluish in color and tense. After durotomy, subdural hematoma with thin membrane was exposed. The hematoma was dark, red wine color and well liquefied, and was evacuated by suction. After normal saline irrigation and bleeding control, watertight dural closure was 


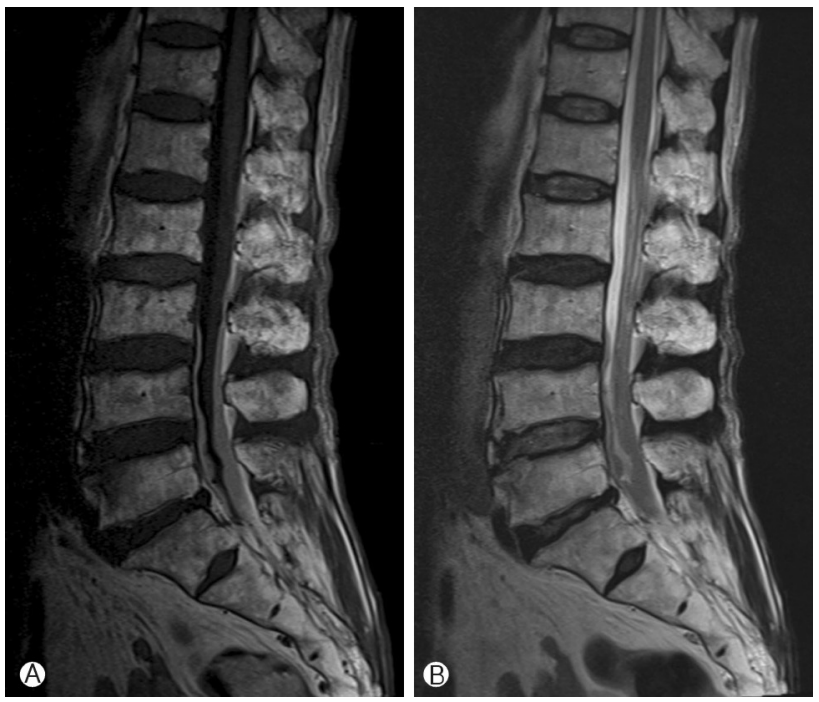

Fig. 1. $\mathrm{Tl}$-weighted image shows an iso-to-hyperintense signal lesion at L2-S2 level (A). T2-weighted image shows an iso-tohypointense signal lesion at L2-S2 level (B).

performed.

Postoperatively, pain in the buttocks and lower extremities were resolved. Two weeks after operation, the patient had no pain and was discharged. A follow-up MRI study performed 2 months after operation, documented no evidence of residual hematoma (Fig. 2). At 1-year-follow up, there was no postoperative complication and the patient had no more pain.

\section{DISCUSSION}

Spinal SDH is remarkably uncommon, which must be diagnosed accurately and promptly. In some cases, compression of the spinal cord by spinal SDH could lead to acute paraplegia and requires treatment before irreversible neurological damage occurs.

Based on causes, idiopathic spinal SDH is rare and in secondary spinal SDH, the most common causes of spinal SDH are coagulopathies, anticoagulant therapy, spinal trauma, or post iatrogenic circumstance ${ }^{7,9,11}$. Other various causes include tumors, ruptured vascular malformation including arteriovenous malformation, or aneurysm of spinal artery ${ }^{5,9,11)}$.

The mechanisms of formation of spinal SDH remain unclear. Various hypotheses of spinal SDH have been proposed, increased intravascular pressure in subdural space due to increased intrathoracic or intraabdominal pressure resulting from trauma, cause a failure in the spinal cord vascular. Another hypothesis is migration of hematoma from intracranial subarachnoid to the spine due to gravity ${ }^{8,13)}$.

MRI provides signal from hematoma changes over time after onset that is similar to those of brain hematoma ${ }^{5,6}$.

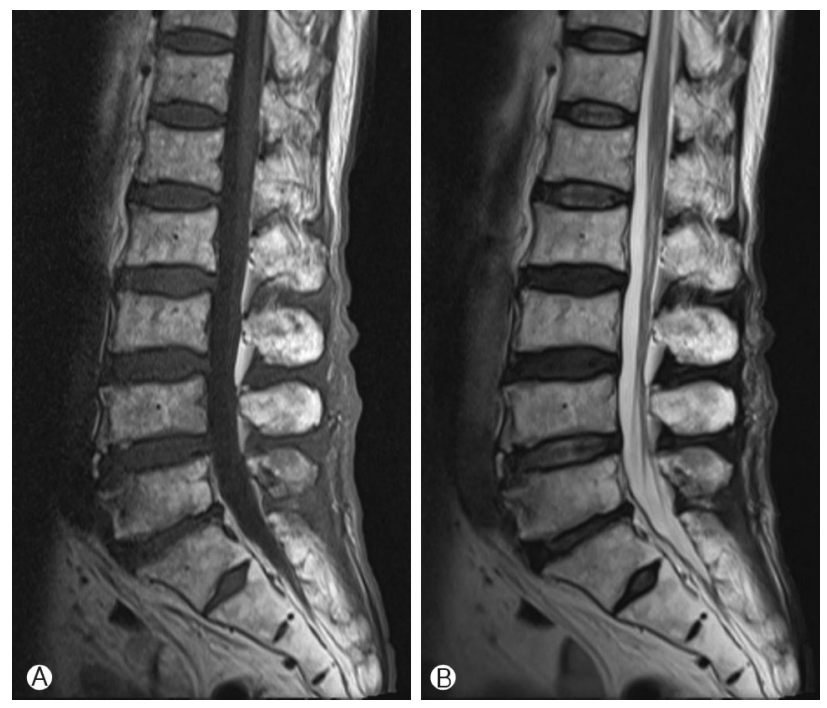

Fig. 2. Postoperative $\mathrm{T} 1$-weighted (A) and T2-weighted (B) images show no residual hematoma.

Immediately after attack, hematoma appearance on T1-weighted image is intermediate signal intensity area and on T2weighted image is low signal intensity. Between 7 to 14 days after attack, hematoma appears as high-to-intermediate signal intensity on T1-weighted image, and an increase in signal intensity on T2-weighted image.

In our case, signal intensity was high-to-intermediate in T1weighted image, and low-to-intermediate image in T2-weighted image. The MRI findings are not typical results of chronic spinal SDH which make preoperative diagnosis difficult. In the operation field, nature of hematoma was much liquefied and was dark, red wine like color. We could suck out hematoma easily and irrigate hematoma completely.

The treatment of choice is laminectomy and drainage of hematoma ${ }^{4,9,13)}$. Despite of spontaneous remission of intraspinal hematoma has been reported, early surgical decompression is commonly required, because of progression of neurological deterioration caused by spinal cord compression, ischemia, and direct injury to spinal cord. Early surgical decompression results in a good outcome. The literature showed 80\% improvement in neurological function after surgery ${ }^{1,3,10,13)}$.

\section{CONCLUSION}

Spinal chronic SDH is rare but should be considered in patients with sudden back pain, radiating pain to the upper or lower extremities or trunk and motor, sensory, autonomic disturbance. In these patients, prompt radiologic assessments, especially MRI, is required. Good outcome can be achieved with early diagnosis and surgical decompression. 


\section{REFERENCES}

1. Abla AA, Oh MY: Spinal chronic subdural hematoma. Neurosurg Clin N Am 11:465-471, 2000

2. Amidei C.: Measurement of physiologic responses to mobilisation in critically ill adults. Intensive Crit Care Nurs 3:2012

3. Brandt RA: Choronic spinal subdural hematoma. Surg Neurol 13:121-123, 1980

4. Dampeer RA: Spontaneous spinal subdural hematoma. Am J Crit Care 19:191-193, 2010

5. Diyora B, Sharma A, Mamidanna R, Kamat L: Chronic cervicothoracic spinal subdural hematoma. Neurol Med Chir (Tokyo) 49(7):310-312, 2009

6. Duprez T, Grandin C, Malghem J: MRI monitoring of an acute spinal subdural hematoma with spontaneous resolution. Acta Neurol Belg 95:101-103, 1995

7. Greiner Perth-R, Mohsen Allam Y, Sibermann J, Gahr R: Traumatic subdural hematoma of the thoraco-lumbar junction of spinal cord. J Spinal Disord Tech 20(3):239-241, 2007

8. Hamaguchi H, Osawa T, Koyama K, Daito M, Yamazoe S, Mikami Y, et al: Idiopathic lumbar spinal subdural hematoma. Orthopedics 31(7):715, 2008

9. Kim MS, Lee CS, Lee SJ, Rhee JJ: Spinal subdural hematoma following intracranial aneurysm surgery: four case reports. Neuro Med Chir 47(1):22-25, 2007

10. Leber KA, Pendl G, Kogler S, Kammerhuber F, Ebner F: Simultaneous spinal and intracranial chronic subdural hematoma: case illustration. J Neurosurg 87:644, 1997

11. Mashiko R, Nohuchi S, Uemura K, Takada T, Matsmura A: Lumbosacral subdural hematoma: case report. Neurol Med Chir 46(5):258-261, 2006

12. Nam Gu Kang, Rae Seop Lee, Kyu Yong Cho: Spontaneous Spinal Subdural Hematoma: Two Case Reports. Korean J Spine 3(2):177-121, 2006

13. Su YF, Hwang YF, Lee KS, Lin CL, Howng SL, Hwang SL: Spinal chronic subdural hematoma in a patient with ventriculoperitoneal shunting after a minor trauma. Spinal Cord 42:717719,2004 